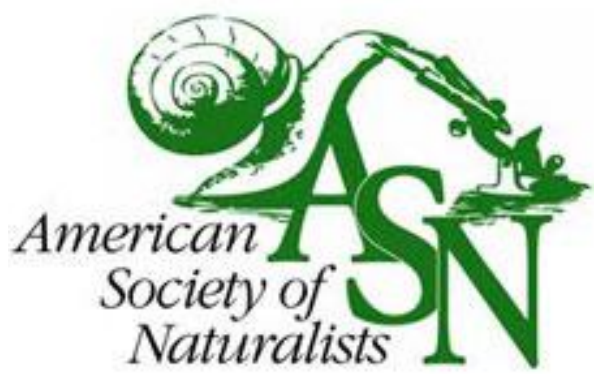

Energetic Constraints on Fungal Growth

Author(s): Luke L. M. Heaton, Nick S. Jones and Mark D. Fricker

Source: The American Naturalist, e American Naturalist (February 2016), p. E000

Published by: University of Chicago Press for American Society of Naturalists

Stable URL: http://www.jstor.org/stable/10.1086/684392

Accessed: 30-12-2015 11:40 UTC

Your use of the JSTOR archive indicates your acceptance of the Terms \& Conditions of Use, available at http://www.jstor.org/page/ info/about/policies/terms.jsp

JSTOR is a not-for-profit service that helps scholars, researchers, and students discover, use, and build upon a wide range of content in a trusted digital archive. We use information technology and tools to increase productivity and facilitate new forms of scholarship. For more information about JSTOR, please contact support@jstor.org. 


\title{
Energetic Constraints on Fungal Growth
}

\author{
Luke L. M. Heaton, ${ }^{1, \star}$ Nick S. Jones, ${ }^{2, \dagger}$ and Mark D. Fricker ${ }^{1,3, \dagger}$ \\ ${ }^{1}$ Department of Plant Sciences, University of Oxford, South Parks Road, Oxford, OX1 3RB, United Kingdom; ${ }^{2}$ Department of Mathematics, \\ Imperial College London, London, SW7 2AZ, United Kingdom; ${ }^{3}$ CABDyN (Complex Agent-Based Dynamic Networks) Complexity \\ Centre, Sad Business School, University of Oxford, Park End Street, Oxford, OX1 1HP, United Kingdom
}

Submitted April 14, 2015; Accepted July 23, 2015; Electronically published December 8, 2015

Online enhancement: appendixes, Matlab code.

\begin{abstract}
A BSTRACT: Saprotrophic fungi are obliged to spend energy on growth, reproduction, and substrate digestion. To understand the trade-offs involved, we developed a model that, for any given growth rate, identifies the strategy that maximizes the fraction of energy that could possibly be spent on reproduction. Our model's predictions of growth rates and bioconversion efficiencies are consistent with empirical findings, and it predicts the optimal investment in reproduction, resource acquisition, and biomass recycling for a given environment and timescale of reproduction. Thus, if the timescale of reproduction is long compared to the time required for the fungus to double in size, the model suggests that the total energy available for reproduction is maximal when a very small fraction of the energy budget is spent on reproduction. The model also suggests that fungi growing on substrates with a high concentration of low-molecular-weight compounds will not benefit from recycling: they should be able to grow more rapidly and allocate more energy to reproduction without recycling. In contrast, recycling offers considerable benefits to fungi growing on recalcitrant substrates, where the individual hyphae are not crowded and the time taken to consume resource is significantly longer than the fungus doubling time.
\end{abstract}

Keywords: energy allocation, fungal growth, autophagy, life-history strategies.

\section{Introduction}

Fungi are ubiquitous and ecologically critical. They are largely responsible for the breakdown of woody debris, and the rate of decomposition has a major impact on the rate of carbon and mineral nutrient cycling in ecosystems (Dighton 2003; Deacon 2005; Boddy et al. 2008). New resources are spatially discontinuous but may be "discovered" by spore dispersal or extensive foraging of mycelium in non-resource-restricted species (Dowson et al. 1986; Boddy 1999; Boddy et al. 2009). Subsequent decomposition involves growth in or on the resource, secretion of digestive enzymes to break down the substrate, and active uptake

* Corresponding author; e-mail: luke.heaton@gmail.com.

${ }^{\dagger}$ N. S. Jones and M. D. Fricker contributed equally to this manuscript.

Am. Nat. 2015. Vol. 187, pp. E000-E000. ㄷ 2015 by The University of Chicago. 0003-0147/2016/18702-56202\$15.00. All rights reserved.

DOI: $10.1086 / 684392$ of the solubilized products to fuel further growth or reproduction (Boddy et al. 2008). Within this overall framework, each fungal species adopts a different strategy, depending on the relative investment in growth, resource acquisition, or reproduction.

To explore the trade-offs between these essential but conflicting patterns of energy expenditure and to understand how different life-history strategies (Andrews 1992) may emerge in the saprotrophic fungal community, we have constructed an energy-budget model. This model has some features in common with classic, dynamic energy-budget models (Kooijman 2009; Sousa et al. 2010), as we consider both stores of energy and the energetic cost of building and maintaining structures, but we constructed our model from first principles appropriate for a fungal system rather than adapting an existing model. This was necessary because fungal colonies do not fit well into existing models for either plants or animals, although they exhibit features that characterize both. In particular, colonization of new terrain through spores is reminiscent of seed dispersal in plants, but local foraging for resources is typical of animals, although in the case of fungi "foraging" involves growth as an interconnected mycelial network rather than movement. Such networks are highly dynamic and have to balance discovery of new resource through exploration with exploitation of existing resource while maintaining transport between changing sources and sinks in the face of predation and competition (Boddy 1999; Bebber et al. 2007; Fricker et al. 2007; Heaton et al. 2012a). Furthermore, fungal growth is highly plastic, and the quality and distribution of resource influence the pattern of mycelial growth (Dowson et al. 1986; Bolton and Boddy 1993; Ritz 1995; Boddy et al. 2009). For example, fungi exploit higher-quality resources by increasing the rate of hyphal branching, producing relatively dense mycelia with larger apparent fractal dimension (Bolton and Boddy 1993; Ritz 1995).

Resource levels also influence the trade-off between growth and reproduction, as larger fungi can produce more spores, and many species require a mycelium of some minimal size before they can grow reproductive structures 
(Plunkett 1953; Madelin 1956). Characteristics of the resource environment help determine how much energy is available for the production of reproductive propagules, but the timing and amount of fruiting also depend on the nutrient composition of the substrate and other environmental factors (Plunkett 1953; Simchen 1966; Suberkropp 1991; Boddy et al. 2014). In addition to consuming substrates, many saprotrophic fungi can recycle redundant parts of the mycelium through processes such as autophagy to recover critical nutrients, such as nitrogen (Deacon 2005; Pollack et al. 2009; Shoji and Craven 2011; Josefsen et al. 2012). However, recycling also has a cost, as it reduces the biomass density of the fungus, compromising its ability to defend territory, tolerate disturbance, or synthesize and transport new cell materials needed for growth (Boddy 1999; Heaton et al. 2012a). Consequently, it is not obvious when recycling will be beneficial or why some species but not others recycle extensively (Falconer et al. 2005).

In an ecological context, it is not clear what behaviors will best promote the survival of offspring relative to the survival rate of other species in the same niche. The impact of different strategies for resource allocation has been explored through models that vary the investment in reproduction as a fraction of total energy use (Schmit 1999, 2002; Gilchrist et al. 2006), but it is remarkably challenging to evaluate the relationship between fungal traits, life-history strategy, and fitness (Pugh 1980; Hamilton et al. 1987; Andrews 1992; Pringle and Taylor 2002). Despite the difficulty of quantifying relative fitness, it is reasonable to assume that fungi are well adapted to the fundamental challenge of finding sufficient energy to fuel their reproductive efforts. For example, it is plausible that species with a higher growth rate will outcompete species with a lower growth rate (all else being equal), as they are expected to capture a larger fraction of the available resource. Similarly, species that convert a larger fraction of the energy sources they consume into reproductive effort will outcompete species that spend less on reproduction per joule of resource consumed, because (all else being equal), they will have the energy budget to produce larger numbers of offspring. However, there is an inherent trade-off between rapid growth and efficient exploitation of captured resource, as energy that is spent on reproduction cannot be spent fueling growth. Our model uses a common energy currency to systematically explore how different rates of growth, recycling, and investment in digestion and resource assimilation affect the amount of energy available for reproduction and how these trade-offs are affected by characteristics of the resource environment.

For each environment and each net growth rate from 0 to the maximum, we can use our model to identify a unique strategy that yields the largest possible fraction of energy available for reproduction (for the given growth rate) Hence, for each environment we identify a range of "opti- mal" strategies (one for each growth rate), ranging from the strategy that exploits the captured resource in the most efficient way possible to the strategy that enables the highest possible growth rate. In this way we can identify how selective pressure for rapid growth or efficient use of captured resource will affect fungal behavior. We can also explore how these "optimal" strategies vary with environmental parameters. We find that for any given rate of growth and recycling, there is some intermediate rate of resource consumption that maximizes the fraction of energy available for reproduction. More rapid growth requires a larger fraction of energy spent on digestive enzymes and transporters to capture resources, and the "optimal" rate of investment in digestion and resource assimilation may approach (but not exceed) half the energy budget. We also find that recycling is beneficial when fungi are growing on substrates that are recalcitrant, patchy, or nutrient poor but that recycling is not beneficial if the substrate enables rapid growth, with significant transport costs.

We do not need to make any assumptions about the relative importance of rapid growth or efficient conversion of captured resource into reproductive effort, but it is plausible that species that expend a larger total amount of energy on reproductive propagules (per individual) will outcompete species that spend less on reproduction, as producing larger numbers of reproductive propagules is likely to result in larger numbers of offspring. This assumption is not necessarily valid under all circumstances and for all fungal species, but it is a good starting point for assessing trade-offs in energy expenditure in a fungal mycelium. In particular, this simplifying assumption reveals the importance of the timescale of reproduction. If a smaller fraction of the energy budget is invested in reproduction, fungi can double in size more rapidly and thereby greatly increase the total amount of resource they encounter. Thus, if the timescale of reproduction is long compared to the time required for the fungus to double in size, the total energy available for reproduction is maximal when only a small fraction of the joules encountered are spent on reproduction. It follows that fungal mycelia utilizing large, durable resources (e.g., dead trees) can be expected to increase the chances of offspring survival if they grow as rapidly as possible, reproduce slowly, and allocate only a small fraction of the available energy to reproduction. Conversely, fungal mycelia utilizing ephemeral resources (e.g., dung) can be expected to increase the chances of offspring survival if they reproduce rapidly and allocate a much larger fraction of the available energy to reproduction.

\section{Model Assumptions}

For the sake of simplicity we consider a single resource (e.g., carbon), which can effectively be measured in terms 
of energy. We suppose that the energy content per unit volume of fungus is a constant, and it is therefore convenient to define a unit volume as that volume of fungus that contains $1 \mathrm{~J}$ of combustible energy (removing the need for a conversion factor between fungal volume and units of energy). We also suppose that each part of the environment either contains resource at some given concentration or does not contain any resource, either because it never contained resource or because the resource has been consumed. It is this simplifying assumption that enables us to construct a model of energy use that involves only two state variables: $V(t)$, which denotes the volume of fungus at time $t$, and $f(t)$, which denotes the fraction of the colony that is in contact with resource at time $t$ (see fig. 1).

Organisms cannot grow without a source of energy, and in our model we consider both external and internal sources. Fungi secrete digestive enzymes that break down complex macromolecules in the environment and release solubilized, low-molecular-weight compounds that are actively accumulated through transport proteins in the plasma membrane (Lindahl and Olsson 2004; Deacon 2005). Thus, we assume that resource is consumed at a rate that is proportional to the amount of energy spent on digestion and resource uptake, eventually leaving a zone where the resource has been depleted. Because of this assumption, our model is appropriate only where the rate of nutrient flux into a region is negligible compared to the rate of resource uptake, so the fungi effectively grow on patches of resource that are totally consumed. Parts of the fungus that are not in contact with resource or have consumed the local resource can be recycled, and we assume that when the fungus is recycled it yields some fraction $\mu$ of its embodied energy.

In our model, fungal growth strategies are described in terms of control parameters. The first control parameter is the specific growth rate $g(t)$, and by assumption, at time $t$ the volume of hyphae that are produced per unit time is $V(t) g(t)$. Fungi are unusual among multicellular organisms in that their overall morphology is highly plastic and redundant elements can be recycled. We therefore include a recycling rate $r(t)$ as a second control parameter. Only parts of the fungus that are not in contact with resource are recycled, but it is mathematically more convenient to let $r(t)$ denote the fraction of the entire fungus that is recycled per unit time. In other words, the volume recycled per unit time is $V(t) r(t)$, and the net rate of volumetric growth is $V(t)(g(t)-r(t))$. Fungi can also vary the amount of energy that is spent on digestive enzymes and transporters, which means they can control the rate at which resource is consumed. For this reason, our model contains a third control parameter, $c(t)$, and we suppose that each unit volume of fungus that is in contact with resource consumes $c(t)$ units of resource per unit time.

We let $s(t)$ denote the rate at which surplus resource is stored per unit volume of fungus, averaged over the entire fungus. It follows that $V(t) s(t)$ units of resource are stored per unit time, and this store of resource represents the en-

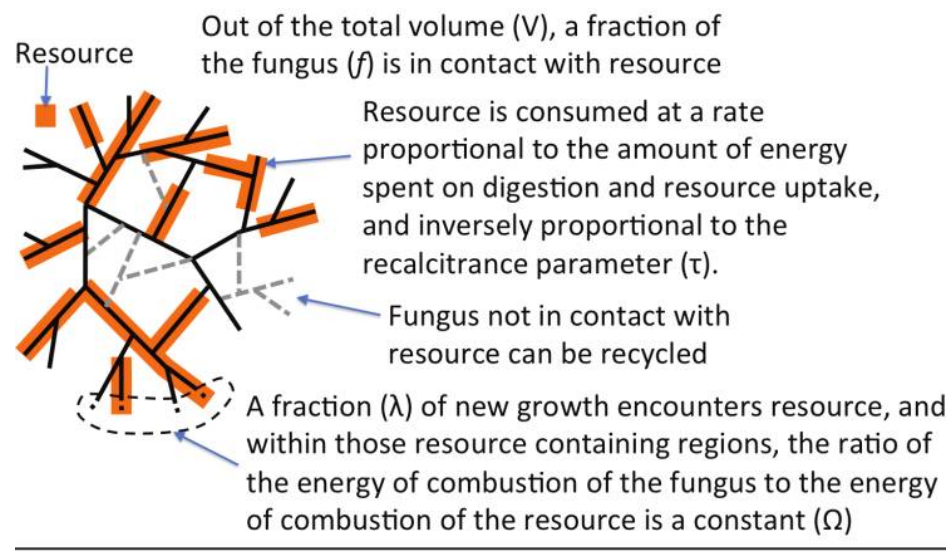

Fundamental Equation (1):

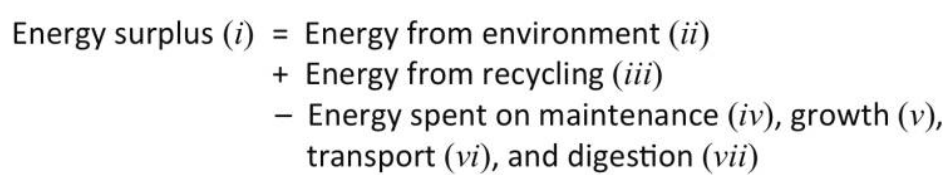

Figure 1: Model of fungal growth. The state of a fungal network at time $t$ is summarized by the volume of the fungus $V(t)$ and the fraction of the fungus in contact with resource $f(t)$. Fungi can control the rate at which they spend energy on growth, digestion, and reproduction, and they can control the rate at which they recycle biomass. These rates will determine both the volumetric growth rate and the fraction of the fungus that is in contact with resource. 
Table 1: Parameters and variables of the model

\begin{tabular}{|c|c|c|}
\hline Parameter or variable & Symbol & Interpretation \\
\hline Maintenance parameter & $\kappa$ & $\begin{array}{l}\text { Fraction of the energy embodied in the colony that is consumed paying maintenance } \\
\text { costs per unit time }\end{array}$ \\
\hline Growth parameter & $\alpha$ & It costs $\alpha$ joules to synthesize the cellular components contained in $1 \mathrm{~J}$ of fungus \\
\hline Transport parameter & $\beta$ & Transport costs are proportional to $\beta$ \\
\hline Recycling efficiency & $\mu$ & Fraction of embodied energy that is recouped when part of the fungal colony is recycled \\
\hline Resource density & $\lambda$ & Probability that a newly formed part of a fungal colony encounters resource \\
\hline Energy ratio & $\Omega_{0}$ & $\begin{array}{l}\text { Total number of joules consumed per joule of fungus when } g(t) \text { is very small and there is } \\
\text { no crowding }\end{array}$ \\
\hline Crowding parameter & $\Upsilon$ & $\begin{array}{l}\text { If the doubling time is much larger than } \Upsilon \text {, crowding effects are negligible; when } \Upsilon g=1 \text {, } \\
\text { the number of joules of consumable resource per unit volume of fungus is halved, } \\
\text { relative to the uncrowded case }\end{array}$ \\
\hline Recalcitrance parameter & $\tau$ & $\begin{array}{l}\text { Time taken to consume a piece of resource if half the parameter energy embodied in the } \\
\text { resource is spent on digestion }\end{array}$ \\
\hline Volume & $V(t)$ & Volume of fungus at time $t$ \\
\hline $\begin{array}{l}\text { Fraction of fungus in contact } \\
\text { with resource }\end{array}$ & $f(t)$ & Fraction at time $t$; under the quasi-steady state assumption, $f(t)$ is a constant $\tilde{f}$ \\
\hline Growth rate & $g(t)$ & Volume of new growth produced per unit volume per unit time \\
\hline Recycling rate & $r(t)$ & Fraction of total fungal volume that is recycled per unit time \\
\hline Resource consumption & $c(t)$ & Energy consumed per unit time by each unit volume of fungus in contact with resource \\
\hline Storage rate & $s(t)$ & Energy stored or spent on reproduction per unit volume per unit time \\
\hline
\end{tabular}

Note: See appendix B, available online, for a discussion of the empirical range of the parameters.

ergy budget available for reproduction or any other process that is not otherwise accounted for in our model, such as production of defensive compounds. Note that although $s$ has the appearance of a fourth control parameter, it is completely determined by the other control parameters, because we assume that at all times the total amount of energy stored is equal to the total amount of energy taken from the environment plus the total amount of energy recouped through recycling, minus the total amount of energy spent on growth, transport, maintenance, and digestion. Because of this assumption, we can calculate $s$, given the state variable $f(t)$ and the three control parameters $g, r$, and $c$.

In addition, there are three fixed parameters that define the costs of fungal behavior, $\kappa, \alpha$, and $\beta$, which, respectively, determine the costs associated with maintenance, growth, and transport, plus three parameters $\lambda, \Omega$, and $\tau$ that describe the resource environment (see fig. 1 and table 1 for a list of all parameters and app. B, available online, for a discussion of the appropriate numerical values for these parameters $\left.{ }^{1}\right)$. More specifically, we assume that a fraction $\lambda$ of newly grown hyphae encounter resource and that each joule of fungus that does encounter resource has access to a total of $\Omega \mathrm{J}$ of resource. Hence, $\lambda$ is related to the density of resource in the environment, and the energy ratio $\Omega$ is re-

\footnotetext{
1 The Matlab code for our model is available online. Code that appears in the American Naturalist is provided as a convenience to the readers. It has not necessarily been tested as part of the peer review.
}

lated to the quality of that resource. The rate at which that resource is consumed will depend on the amount of energy that is spent on digestive enzymes and transporters, but clearly some substrates are easier to consume than others. For example, given the same investment in digestion and resource uptake, $1 \mathrm{~J}$ of nutrient agar will be consumed much more rapidly than $1 \mathrm{~J}$ of wood. In our model, the recalcitrance of the substrate is captured by a parameter $\tau$, which can be interpreted as the shortest time in which the resource could possibly be consumed (see "Digestion Costs" in app. A, available online).

Finally, we note that high growth rates will tend to produce crowded fungal colonies where individual hyphae compete with one another for the surrounding resource. Hence, the energy ratio $\Omega$ is actually a function of the growth rate $g$ and not a simple parameter. We model this crowding effect with a logistic equation (Papagianni 2004; Fathima et al. 2013) and therefore require an additional "crowding parameter" $\Upsilon$ to specify the extent of such growth-rate-dependent crowding effects.

\section{Fundamental Model Equations}

In the fundamental equation (1), we assume that the rate at which energy is stored or used for reproduction (i) is equal to the rate at which resource is consumed (ii) plus the rate at which energy is obtained by recycling (iii), minus the rate at which energy is spent on maintenance (iv), 
growth (v), transport (vi), and digestion (vii), where the time dependence of $V, f, g, r, c$, and $s$ have been dropped for clarity:

$$
\begin{aligned}
\overbrace{V s}^{(\mathrm{i})}= & \overbrace{V f c}^{(\mathrm{ii})}+\overbrace{V \mu r}^{(\mathrm{iii})}-\overbrace{V \kappa}^{\text {(iv) }}-\overbrace{V \alpha g}^{(\mathrm{v})} \\
& -\underbrace{V \beta \frac{f-2 f \lambda+\lambda^{2}}{f(1-f)} g^{2}}_{\text {(vi) }}-\underbrace{V \frac{f c^{2} \tau\left(\Upsilon_{g}+1\right)}{2 \Omega_{0}}}_{\text {(vii) }} .
\end{aligned}
$$

Each term is a multiple of the volume $V$, so the relationship between $f, g, r, c$, and $s$ does not depend on the size of the colony $V$ per se. Also note that term i follows by definition, as $s$ denotes the rate at which energy is saved per unit volume of fungus. The total volume of fungus in contact with resource is $V f$, and each of those unit volumes consumes resource at a rate $c$. Hence, term ii, the total rate of resource consumption, is $V f c$. The volume recycled per unit time is $V r$, and we assume that each unit volume of fungus that is recycled yields $\mu$ units of energy. Hence, term iii, the supply of energy from recycling, is $V \mu r$.

The remaining terms in equation (1) represent the costs of maintenance, growth, transport, and digestion. Term iv is $V \kappa$, as we have assumed that the maintenance costs are proportional to the fungal volume. We also investigated a model in which parts of the mycelium that are not in contact with resource become quiescent, with no metabolic costs for maintenance. This alternate model produced very similar results, except that the optimal rate of recycling is reduced, because recycling quiescent hyphae does not help to reduce maintenance costs. The "growth cost" (v) accounts for the metabolic cost of synthesizing the cellular constituents that will occupy the newly formed parts of the colony, as well as any other costs that are proportional to the rate of growth. The cost of synthesizing a new unit volume is $\alpha$, so when biomass is produced at a rate $V g$, the total cost per unit time due to growth is $V \alpha g$.

The metabolic cost of growth is more than just the cost of protein synthesis, as materials must be transported from the site of uptake through the mycelial network to the growing margin, where vesicles containing cell-wall materials fuse with the growing tips (Wessels 1993; Lindahl and Olsson 2004; Steinberg 2006; Money 2008). We suppose that the total cost of transporting a unit volume is proportional to the speed of transport, and the transport cost (vi) is derived from this and other considerations (see "Transport Costs" in app. A). Finally, we need to account for the cost of digestion (vii), which is done by assuming that the rate of resource consumption is proportional to the amount of energy invested in the digestive process (see "Digestion Costs" in app. A).

Equation (1) shows that the growth rate $g$, the recycling rate $r$, and the resource consumption rate $c$ determine the rate of resource storage $s$, but the control parameters also determine how the state variables $V$ and $f$ vary over time. In particular, fungal biomass is increased by growth and decreased by recycling, so we have

$$
\dot{V}=V(g-r) \text {. }
$$

Growth increases the volume of fungus in contact with resource, while the consumption of resource turns biomass that was in contact with resource into biomass that is not in contact with resource. We let $W(t)=f(t) V(t)$ denote the volume of fungus in contact with resource and note that the rate of production of fungal volume in contact with resource is $\lambda V g$. Furthermore, since each unit volume in contact with resource consumes a total of $\Omega \mathrm{J}$ at a rate $c$, the process of resource consumption takes a period of time $t=\Omega / c$. It follows that

$$
\dot{W}=\lambda V g-\frac{W c}{\Omega}=V\left(\lambda g-\frac{f c}{\Omega}\right),
$$

which implies that

$$
\dot{f}=(\lambda-f) g+f r-\frac{f c}{\Omega} .
$$

Note that if we hold the control parameters $g, r$, and $c$ constant, equation (2) implies that the volume will grow exponentially with

$$
V(t)=V(0) e^{(g-r) t},
$$

while the fraction of the colony in contact with resource $f(t)$ will vary according to equation (4). If $\dot{f}<0$ and $f(t)$ decreases over time, equation (1) tells us that the rate of resource storage $s$ will also decrease, and it may even become negative, which would indicate that the chosen values of $g$, $r$, and $c$ cannot be sustained. Conversely, if $f(t)$ increases, the rate of resource storage $s$ will also increase.

As a means of dimensional reduction, it is convenient to compare different growth strategies by assuming that in each case the control parameters are constant, following a period where they might have been adjusted, and that the fraction of the colony in contact with resource has obtained a steady state $\tilde{f}$. In other words, in the following analysis we assume that $g, r, c$, and $s$ are all constant and that $f(t)=\tilde{f}$ for all $t$. Note that this assumption is appropriate only when the fungus is continually finding new resource. In the case of fungi that are restricted to a single patch of resource, the quasi-steady state assumption may hold over the timescale required to fully colonize the patch, but it will not hold over the entire life span of the patch, as over that timescale the fungus will degrade the quality of its resource environment. Also note that fixing $g, r$, and $c$ uniquely determines $\tilde{f}$, because equation (4) implies that when $\dot{f}=0$, we must have

$$
\tilde{f}=\frac{\Omega \lambda g}{\Omega(g-r)+c} .
$$




\section{Results A: General Insights}

Costs and Benefits of Recycling Depend on the Environment

Recycling is a major feature of many saprotrophic fungi (Falconer et al. 2005). Recycling redundant mycelium provides an additional source of energy, reduces the cost of maintenance, and increases the proportion of fungus in contact with resource, allowing for a greater supply of energy per unit volume. On the other hand, recycling reduces the biomass density and so decreases the fungus's capacity to synthesize and transport new cellular components. Hence, it is not obvious what level of recycling (if any) will enable the most rapid growth. Our model can be used to address this question. In particular, for any given set of parameter values, we can systematically identify the maximum possible net specific growth rate $g-r$.

Having identified the strategy that maximizes the net specific growth rate, we can calculate the corresponding relative recycling rate $\theta=r / g$, which is the volume recycled per unit volume of growth when the fungus is growing as fast as possible (see fig. 2). We find that fungi should recycle biomass only when they are growing on substrates that are recalcitrant ( $\tau$ is long; fig. $2 a$ ), patchy ( $\lambda$ is small; fig. $2 b$ ), or nutrient poor ( $\Omega$ is low; fig. $2 c$ ). We also find that as we increase the number of joules recouped per joule recycled $\mu$, the optimal amount of recycling increases (see fig. $2 d$ ). However, even when the recycling efficiency $\mu=0$, recycling can be beneficial for growth on nutrient-poor or highly recalcitrant substrates, because it reduces the cost of maintenance and increases the proportion of fungus in contact with resource.

Note that when $\lambda$ is large, most of the fungus is in contact with resource and cannot be recycled, which is why the optimal recycling rate is lower when $\lambda$ is large (see fig. $2 b$ ). Also note that crowding effects and large transport costs increase the cost of growing at rate $g+r$ while recycling at a rate $r$, relative to the cost of growing at rate $g$. Hence, recycling is not beneficial if increasing the growth rate will significantly increase the amount of crowding $(\Upsilon$ is large; fig. $2 e)$ or if the transport costs are very high ( $\beta$ is high; fig. $2 f)$. We therefore expect to see extensive recycling only when the resource environment is challenging and the hyphae are not crowded.

\section{Optimal Rates of Resource Consumption}

Within our model, we can explore what happens if we vary the consumption rate $c$ when the growth rate $g$ and recycling rate $r$ are fixed. If the fungus spends too little on digestion and so $c$ is low, then the supply of energy will not be great enough to grow at the given rate $g$. If $c$ is increased, it reaches the point where the supply of energy is just sufficient to grow at rate $g$, but there is no excess en- ergy to be stored. If $c$ is increased further, the fraction of energy that is available for reproduction also increases, but only up to a certain point. Any investment in digestive enzymes beyond that point is a waste of energy (given that we are considering the case where the growth rate $g$ is fixed). In figure 3, we show planes within the threedimensional control parameter space $(g, r, c)$ that illustrate the fraction of energy consumed that is available for reproduction. Hence, for any given growth rate $g$ and recycling rate $r$, there is some intermediate resource consumption rate $c$ that maximizes the fraction of energy available for reproduction. This "optimal" investment in digestive enzymes and transporters maximizes the total amount of energy available for reproduction, and in the case where growth rates are maximal, this optimal investment in digestion may approach (but not exceed) half the energy budget (see fig. 3).

\section{Exploration and Exploitation: Trade-Offs and Optimal Control}

All else being equal, species with a higher growth rate are expected to outcompete species with a lower growth rate, as they are likely to capture a larger fraction of the available resource, giving higher fitness as a result of more efficient exploration of the resource environment. An equally reasonable claim is that, all else being equal, species that convert a larger fraction of the energy sources they consume into reproductive effort will outcompete species that have less energy to spend on reproduction per joule of resource consumed, giving higher fitness as a result of more efficient exploitation of the resource consumed. In other words, rapid growth and efficient exploitation of captured resource both contribute to fitness, and this should be reflected in the behavior of fungal mycelia. These two goals are apparently in conflict, as energy that is spent on reproduction cannot be spent fueling growth. However, it is plausible that for any given net specific growth rate, fungi recycle themselves and spend energy on digestion and resource uptake in a manner that maximizes the fraction of energy that is available for reproduction. Conversely, if a given fraction of the energy budget is spent on reproduction, it is plausible that fungi should recycle themselves and spend energy on digestive enzymes in a manner that maximizes the net specific growth rate. Hence, without making any assumptions about the relative importance of rapid growth and efficient exploitation of captured resource, we can identify values for the control parameters that maximize exploitation efficiency for a given net growth rate or maximize the net growth rate for a given exploitation efficiency.

This approach produces a range of "optimal" growth strategies, with a unique optimal strategy for each net growth rate from 0 to the maximum. At one extreme, 

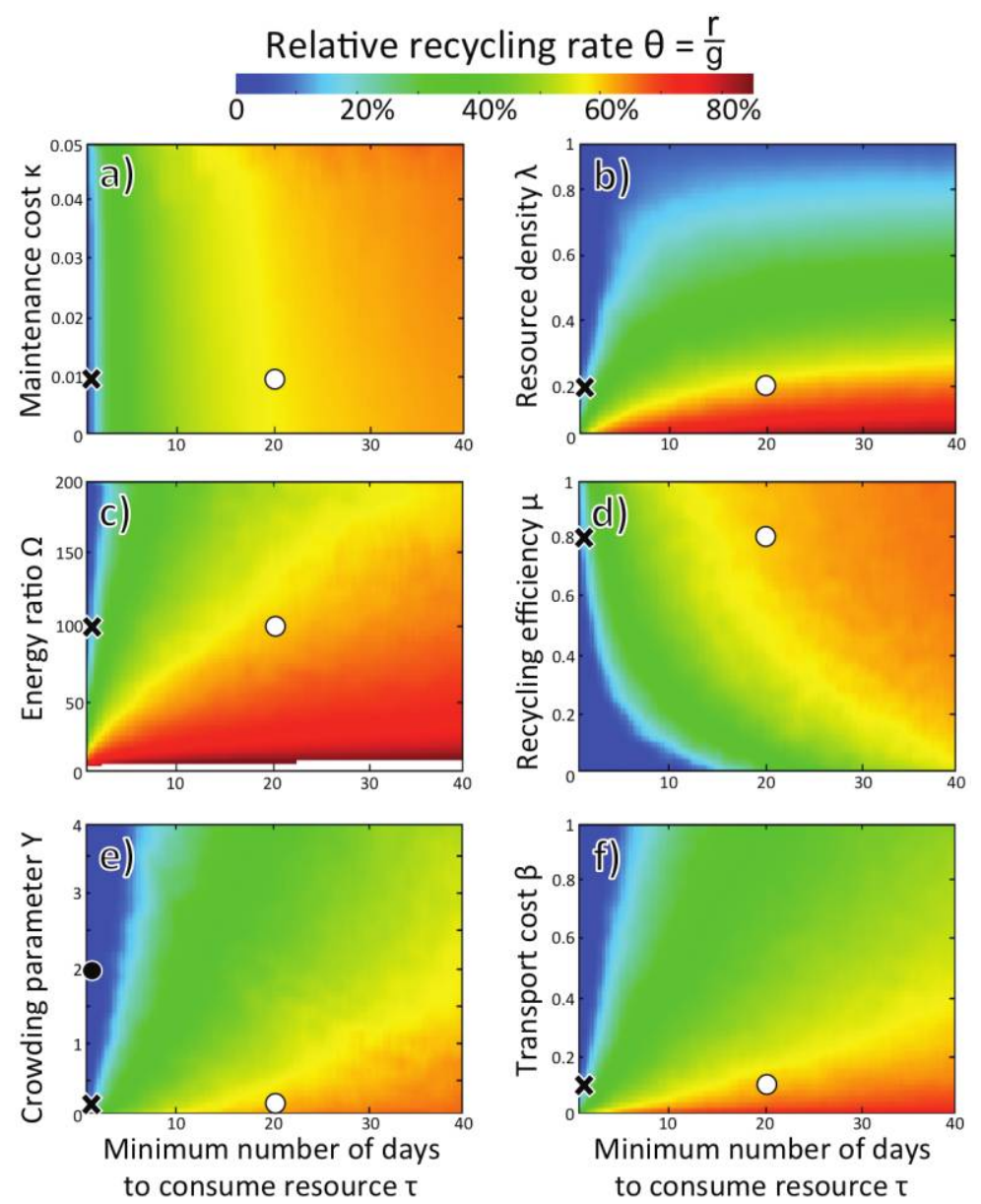

Figure 2: Recycling rate is environmentally sensitive: optimal recycling rates vary with environmental parameters. For each point in parameter space we choose a growth rate $g$, a recycling rate $r$, and a consumption rate $c$ such that the net growth rate $g-r$ is as high as possible, subject to the constraint that the rate of energy consumption must not exceed the rate of energy acquisition. Each heat map represents a slice through the complete, eight-dimensional parameter space $(\tau, \kappa, \lambda, \Omega, \mu, \Upsilon, \alpha$, and $\beta)$, and the color of the heat map represents the relative recycling rate $\theta=r / g$, which is the volume recycled per unit volume of growth when the fungus is growing with the maximum possible net specific growth rate. In each panel the white circle corresponds to the point in parameter space chosen to represent the growth of a fungus foraging for a recalcitrant substrate such as wood. The black cross corresponds to a point in parameter space with the same parameter values, except that in this case the substrate can be assimilated in 1 day rather than 20. The black circle in $e$ represents the point in parameter space chosen to represent growth on nutrient-rich agar, which differs from the case of growth on wood in that the substrate can be assimilated in 1 day and crowding effects are significant. The black circle appears in only one plot because the other slices through parameter space do not pass through the point corresponding to growth on agar (see app. B, available online, for a list of parameter values for growth on wood and agar).

where efficient exploitation is the only concern, we find that whether the growth is occurring on a recalcitrant substrate (wood) or an easily assimilated resource (nutrientrich agar), a strategy of setting $r=g$ so the net growth rate is 0 and investing a small amount of energy in digestive enzymes yields the largest possible fraction of energy available for reproduction. This "optimal" strategy would, in practice, be suboptimal if there were a fitness benefit to more rapid growth, as would be the case if there were competition over resource, with more rapidly growing competitors capturing a larger proportion of the available resource (Andrews 1992; Schmit 1999, 2002; Pringle and
Taylor 2002). If increasing the net growth rate at the cost of exploitation efficiency results in higher fitness, then a smaller fraction of the energy budget should be spent on reproduction, to enable more rapid growth. We find that optimal growth strategies with larger net growth rates involve less recycling per unit volume of growth, and in order to fuel the more rapid growth, these strategies require a larger investment in digestive enzymes (see fig. 4). Furthermore, unlike strategies for maximizing reproductive effort per joule consumed, strategies for producing rapid growth vary considerably with the characteristics of the resource environment. 


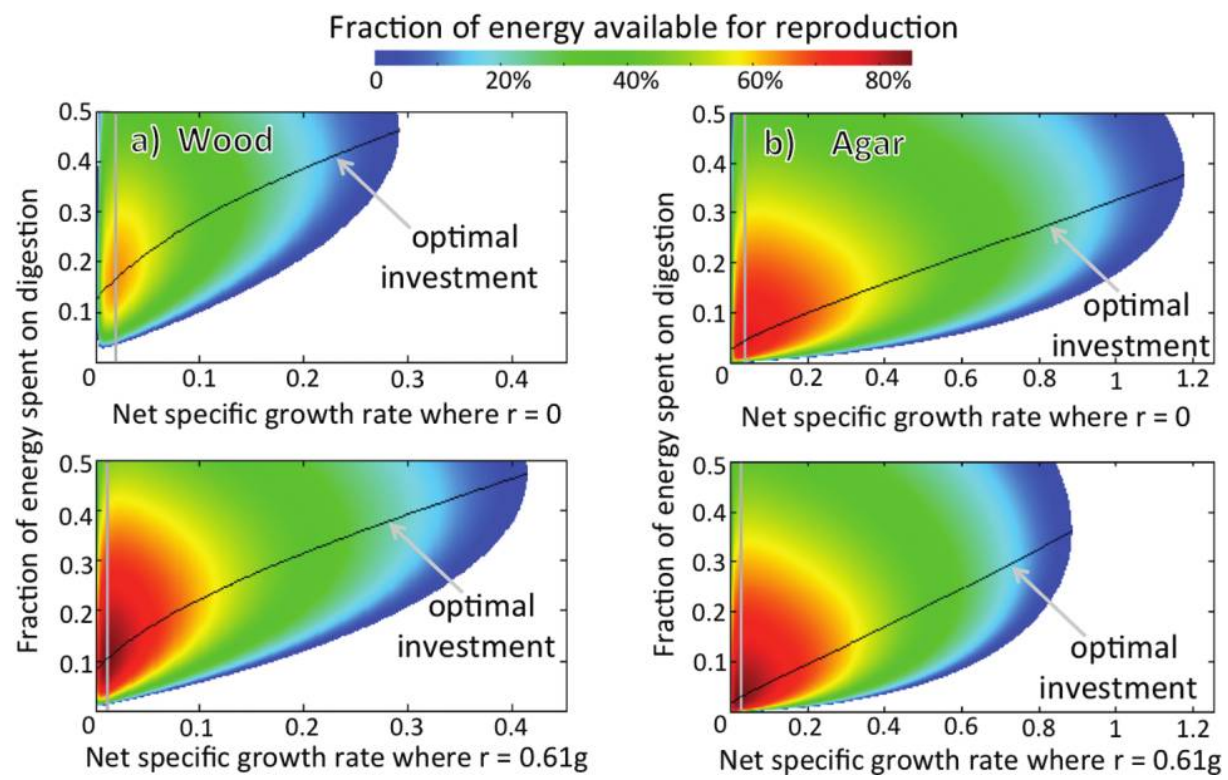

Figure 3: For any given growth rate and recycling rate, the fraction of energy available for reproduction is maximal at a nonmaximal consumption rate $c$. The location of each pixel represents a set of values for the three control variables $g, r$, and $c$, and if these values yield a rate of resource acquisition that is at least as high as the rate of resource consumption, the pixel is colored according to the size of that excess (so the color of pixels represents the fraction of energy consumed that is available for reproduction). The black lines represent the optimal investment in digestion and resource uptake for a given growth rate and a given ratio between $g$ and $r$ (i.e., the investment in digestion that maximizes the fraction of energy available for reproduction). Note that each image represents a slice through a three-dimensional controlparameter space $(g, r, c)$, and we have chosen to illustrate the cases where $r=0$ and $r=0.61 g$, as these are the recycling rates that enable maximal growth on agar and wood, respectively. Also note that for a given ratio between $g$ and $r$, it is counterproductive to grow slower than the rate marked by the gray line, as by altering the control variables the fungus could both grow faster and spend a larger fraction of its energy budget on reproduction.

For the parameter values chosen to represent growth on wood (see app. B), a significant amount of recycling is optimal whatever fraction of energy is saved for reproduction (see fig. $4 a$ ), and the optimal strategies produce colonies in which $\tilde{f}>\lambda$, which means that a larger fraction of the fungus is in contact with resource than would be the case for randomly located hyphae. In the case of growth on agar, recycling is ill-advised even when as much as $38 \%$ of the energy budget is available for reproduction, so most optimal growth strategies produce colonies where $\tilde{f}<\lambda$ (see fig. $4 b$ ). The fact that the fraction $\tilde{f}$ varies with the environmental parameters is also significant because parts of many fungi become quiescent (Deacon 2005), but only if they are not in contact with consumable resource. Hence, the fraction of the fungus that is metabolically active must be at least $\tilde{f}$, and our model leads us to expect a larger amount of potentially quiescent mycelium in cases where the resource is patchy ( small $\lambda$ ) but nutrient rich (high $\Omega$ ) or easily consumed (short $\tau$ ), as such substrates enable rapid growth and rapid growth incurs relatively high transport costs that can be mitigated by maintaining quiescent transport pathways.

\section{Strategies for Maximizing Reproductive Effort Depend on the Timescale of Reproduction}

To identify the relative importance of efficient exploration and efficient exploitation and the "optimal" investment in reproduction, we assume that the total energy spent on reproductive propagules is a key driving force in the evolution of fungal species. This assumption is not necessarily valid under all circumstances and for all fungal species, but is a good starting point for assessing trade-offs in energy expenditure in a fungal mycelium. We note that for a fungus growing on a finite patch of resource, the relative benefit of investing in vegetative growth or production of reproductive propagules will depend on the amount of resource remaining in the patch, the probability per unit time that the patch will be destroyed by a disturbance event, the probability that any given spore will encounter resource and successfully germinate, and the mean amount of new resource captured by successfully germinating spores (Gilchrist et al. 2006).

The size and expected life span of patches of resource will influence the optimal timescale of reproduction (Gil- 

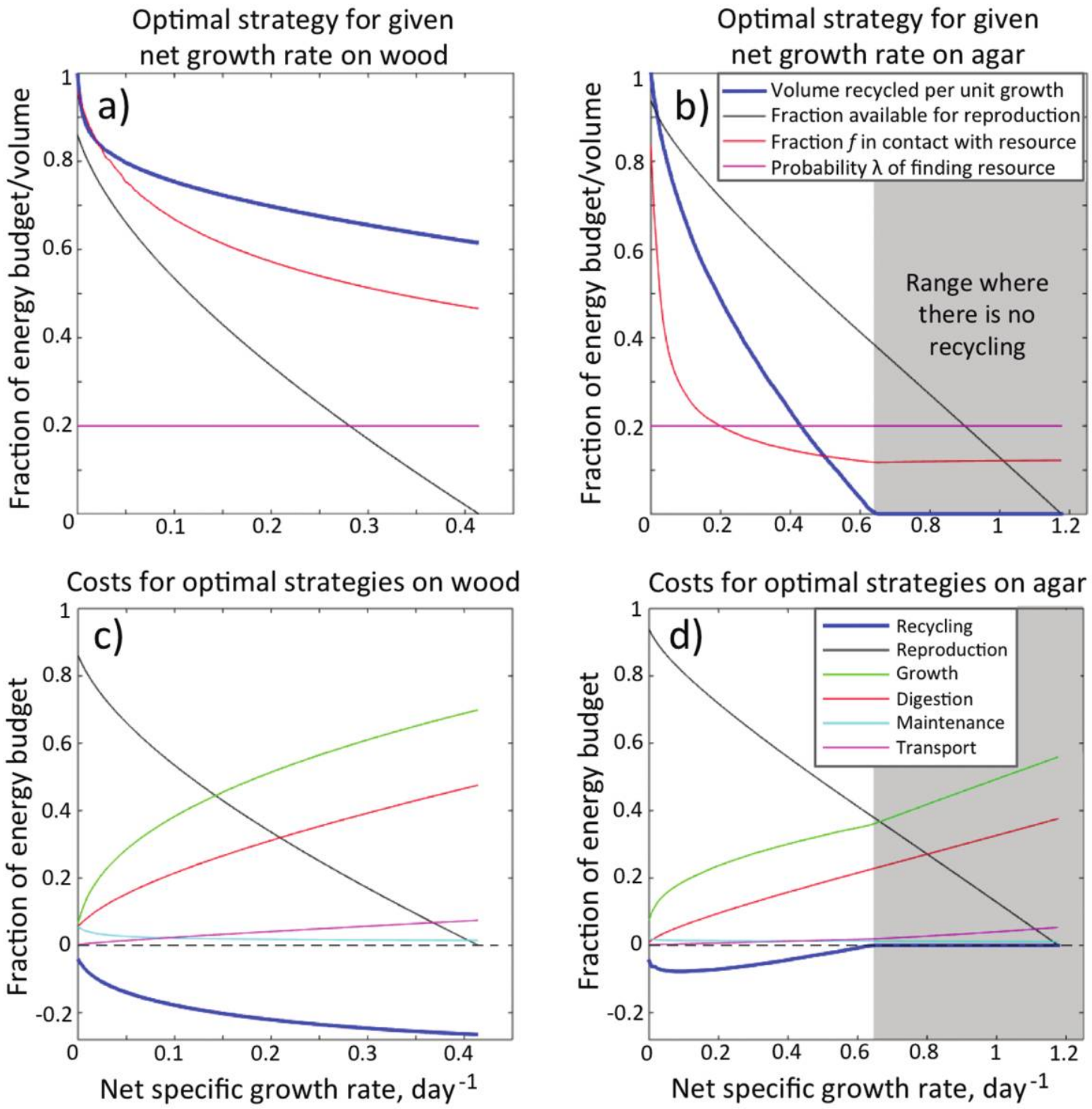

Figure 4: Zero recycling can be optimal for growth on nutrient-rich agar but not for growth on wood. For each net growth rate there is an associated optimal strategy that maximizes the fraction of energy that is available for reproduction. Panels $a$ and $b$ show how the volume recycled per unit growth, the fraction of the colony in contact with resource, and the fraction of energy available for reproduction all decrease as we consider optimal strategies with increasingly high net growth rates. Note that in the case of growth on wood (a), fungi benefit from recycling regardless of net growth rate but that fungi growing on agar $(b)$ should recycle only if they are spending more than $38 \%$ of their energy budget on reproduction. Panels $c$ and $d$ further illustrate the optimal growth strategies for these two media, showing the fractions of energy spent on growth, digestion, maintenance, and transport in each case and the amount of energy gained from recycling relative to the energy gained from external sources.

christ et al. 2006), and this timescale is a critical factor in determining the strategy that will maximize the energy available for the production of reproductive propagules. If the resource that the fungus consumes occurs in small, ephemeral patches (e.g., dung, fruit, or exposed sap), then reproductive structures must be produced rapidly before the substrate is exhausted. Other species grow on relatively persistent, long-lived substrates (e.g., dead trees) and pro- duce fruiting bodies on an annual or biannual basis (Deacon 2005). This difference in the timescale of reproduction will affect the optimal growth strategy (i.e., the strategy that maximizes the total energy available for reproduction) in a manner that can be investigated by our model.

We assume that fungi encounter resource at a rate $\lambda \Omega g V(t)$ and that a fraction $s /(\tilde{f} c)$ of that energy can be spent on reproduction. If we compare strategies by com- 
paring fungi with the same initial volume, letting $V(0)=1$, then it follows that over time $t$ the total amount of energy that is available for reproduction will be

$$
\int_{0}^{t} \frac{\lambda \Omega g s}{\tilde{f} c} V\left(t^{\prime}\right) d t^{\prime}=\frac{\lambda \Omega g s}{\tilde{f} c(g-r)}\left(e^{(g-r) t}-1\right) .
$$

Crucially, the total amount of resource captured by the fungus over time $t$ is proportional to $e^{(g-r) t}$, and this is the only term that increases exponentially. Consequently, when the timescale of reproduction $t$ is long compared to $1 /(g-r)$, a small increase in $g-r$ will result in a large increase in the total pool of resource captured by the fungus. Since investment in reproduction necessarily reduces the energy available for growth, it follows that when the timescale of reproduction is long compared to the time required for the fungus to double in size, only a small fraction of the energy should be spent on reproduction (see fig. 5). However, if the fungus is on an ephemeral resource, it must reproduce rapidly, and so a much larger fraction of the available energy should be spent on reproduction.

We assume that the fraction of new growth that encounters resource is a constant $\lambda$, and this simplification is critical in predicting the optimal fraction of energy to invest in reproduction. This assumption is reasonable while growth is exponential, but for any finite pool of resource, exponential growth can be sustained only for a finite period of time. Once there is no more resource to encounter, the total investment in reproduction would be maximized by halting growth altogether and investing as much energy as possible in reproduction (Plunkett 1953; Morton 1961; Ritz 1995; Gilchrist et al. 2006). On the other hand, if there is competition for a finite patch of resource, more rapid growth should result in the capture of a larger fraction of that finite pool (Schmit 2002). Our model indicates that rapid reproduction should be associated with a relatively large investment in reproduction (as a fraction of total energy). However, as our model currently considers only a single individual, it cannot yet identify the optimal fraction to invest in reproduction during competition over limited resources.

\section{Results B: Comparisons with Empirical Findings}

The model contains five parameters describing the fungus $(\kappa, \alpha, \beta, \mu$, and $\Upsilon)$ and three parameters describing the resource environment $\left(\lambda, \Omega_{0}\right.$, and $\left.\tau\right)$. However, since the parameters are physically interpretable, there are significant constraints on plausible parameter values (see app. B). Furthermore, our choice of parameter values determines both a maximum growth rate and a bioconversion efficiency (that is, joules grown per joule consumed) that can be tested against empirical values. For the parameters chosen to illustrate growth on wood (see app. B), our model predicts a maximal net specific growth rate of $0.41 \mathrm{day}^{-1}$. This compares favorably to the specific growth rate of Articulospora tetracladia grown on submerged ash leaves (0.4-0.7 day $^{-1}$; Gessner $1997 a, 1997 b)$. For nutrient-rich agar, our model predicts a maximal net specific growth rate of 1.2 day $^{-1}$, which compares favorably to the specific growth rates of 0.9 day $^{-1}$ for Marasmius androsaceus, 1.0 day $^{-1}$ for Cryptoporus volvatus, $1.1 \mathrm{day}^{-1}$ for Trichaptum abietinus, and $1.6 \mathrm{day}^{-1}$ for Heterobasidion annosum when these species are grown on nutrient-rich agar plates (Henn et al. 2002). Finally, when fungi are grown in liquid cultures where the colony density is low enough that crowding does not occur, specific growth rates can be as high as 2.9 day $^{-1}$ for Aspergillus niger or 8.4 day $^{-1}$ for Neurospora crassa (Trinci 1971; Deacon 2005). This is in good agreement with our model for nutrient-rich agar when the crowding parameter $\Upsilon=0$, which gives a maximum net specific growth rate of $4.8 \mathrm{day}^{-1}$.

Our model also makes predictions for the bioconversion efficiency, or joules of fungal biomass produced per joule of resource consumed. At the maximum possible growth rate we find a bioconversion efficiency of $43 \%$ for nutrient-rich agar and $21 \%$ for wood. This is comparable to empirical findings, which range from $60 \%$ for $A$. niger growing on low-molecular-weight substrates (Deacon 2005) to $24 \%-$ $46 \%$ for aquatic hyphomycetes decomposing leaf litter (Gessner 1997a, 1997b; Suberkropp 1991), 22\%-40\% for the commercially cultivated Pleurotus tuber-regium (Wu et al. 2003), and $4 \%-21 \%$ for various wood decay fungi (Jones and Worrall 1995).

Another significant implication of our analysis is that if the rate of resource consumption is proportional to the rate at which energy is spent on digestion and uptake, then "digestion costs" should account for a large fraction of the total energy budget. Indeed, in the case where only a small fraction of the energy is spent on reproduction, our model suggests that almost half the energy budget should be spent on digestion and resource uptake (see fig. $4 c, 4 d$ ). We believe that this prediction is plausible, as the "digestion costs" include the running costs of the adenosine triphosphatase and proton-coupled symports that are needed for resource uptake as well as the energetic costs of synthesizing digestive enzymes and transporters. Interestingly, certain strains of Trichoderma reesi excrete more than half of all synthesized proteins into the medium in which they are growing (Knowles et al. 1987; Wessels 1993), which suggests that their investment in uptake and digestion may well represent half their total energy budget.

Finally, we note that our model can predict the optimal investment in reproduction. On nutrient-rich media, the total energy available for reproduction is maximized over a period of 1 day when $51 \%$ of the energy budget is used 

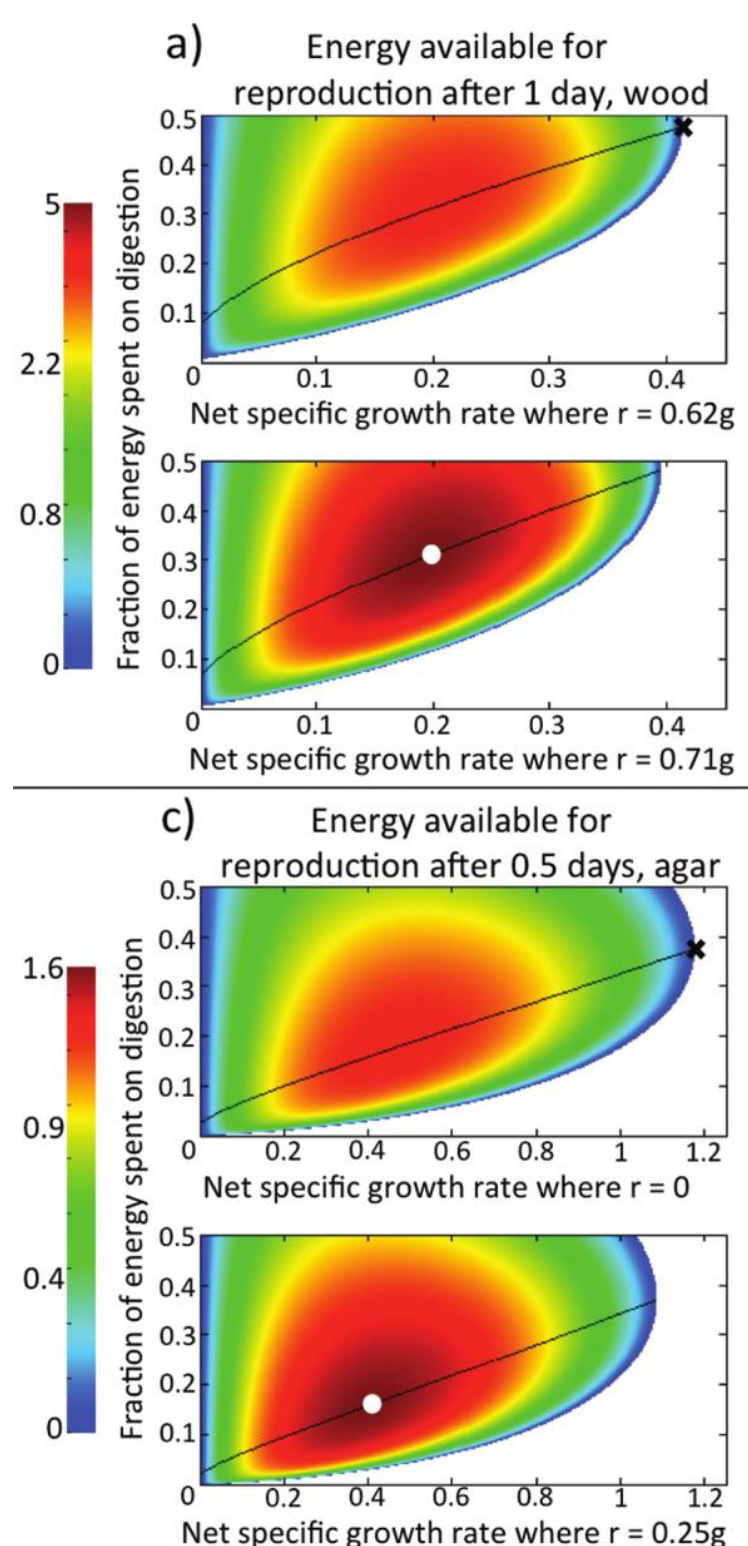

b) Energy available for reproduction after 10 weeks, wood

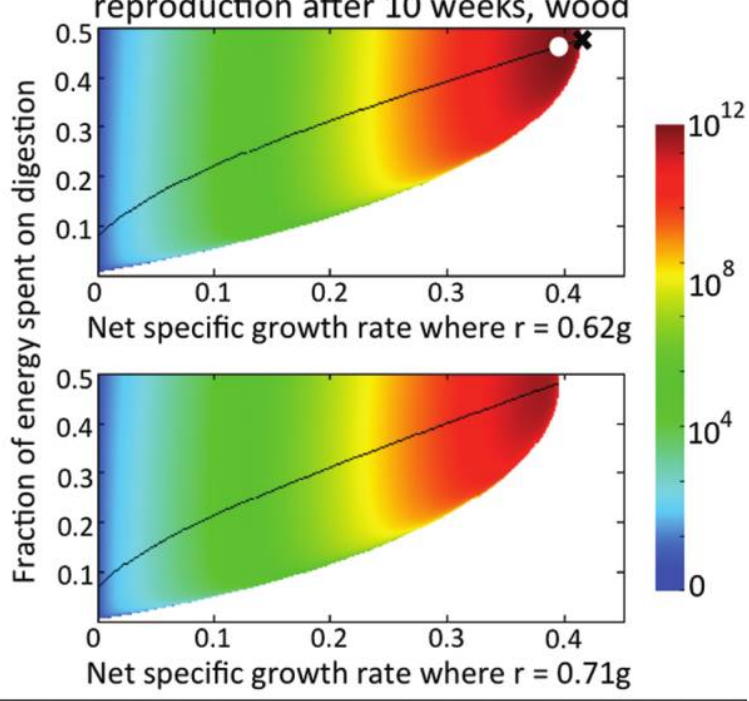

d) Energy available for reproduction after 1 week, agar

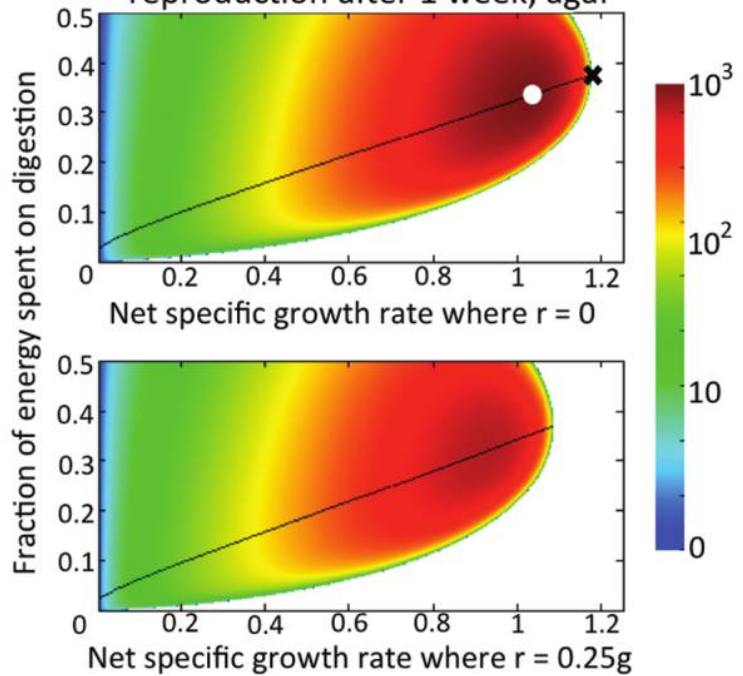

Figure 5: As the timescale for reproduction lengthens, the strategy that maximizes total energy for reproduction (white circle) resembles the strategy to maximize growth rate (black cross). Colors of the heat map indicate the total energy available for reproduction after a given period of time, where energy is measured in units such that one unit of combustible energy is embodied in the colony at time 0 . Panels $a$ and $b$ illustrate the case of growth on a recalcitrant substrate (wood) over a period of time $t=1$ day $(a)$ or $t=10$ weeks $(b)$. Within the threedimensional control-parameter space $(g, r, c)$, we have shown the planes where $r=0.62 g$ and $0.71 g$, as these rates of recycling are optimal over 10 weeks and 1 day, respectively. Panels $c$ and $d$ illustrate the case of growth on an easily assimilated resource (agar) over a period of time $t=0.5$ days $(c)$ or $t=1$ week $(d)$. We have shown the planes where $r=0$ and $0.25 g$, as these rates of recycling are optimal over 1 week and 0.5 days, respectively. As in figure 3, for any given value of $g$ and $r$, the black line indicates the rate of spending on digestion that maximizes the fraction of energy spent on reproduction. Note that in all cases the strategy that maximizes the total energy available for reproduction is located somewhere along the black line, with longer timescales of reproduction favoring less recycling and higher growth rates. Also note that the maximum amount of energy that could be used for reproduction varies enormously between the different cases, as we are considering exponential growth over significantly different timescales. 
for reproduction, exemplified by species such as Neurospora crassa, dropping to $10 \%$ if the time horizon increases to 1 week, more typical of dung fungi (Madelin 1956). Conversely, for growth on wood, the total energy available for reproduction is maximized over a period of 20 weeks with only $1 \%$ investment in reproduction. Twenty weeks is quite typical for the time between fruitings of a wood-decay fungus, although the time to fruiting is highly dependent on seasonality and the impact of climate change (Boddy et al. 2014). Measuring the rate at which fungi allocate energy to reproduction is challenging, as stored resources are difficult to distinguish from the energy embodied in the vegetative mycelium. Furthermore, measurements of the dry weight of reproductive structures as a fraction of total dry weight do not account for energy spent on digestion, maintenance, or transport. Nevertheless, as reproductive structures can account for more than half the dry weight of some species of fungi (Plunkett 1953; Madelin 1956; Suberkropp 1991; Gessner 1997a, 1997b; Schmit 2002), it is clear that rapidly reproducing fungi invest a substantial fraction of their total energy budget on reproductive structures. We could not find equivalent figures for species that reproduce over timescales much longer than their doubling time, but our model predicts that a much smaller fraction of the energy available should be spent on reproduction.

We also note that experiments with the commercial button mushroom Agaricus bisporus and the white rot fungus Schizophyllum commune have found that strains that reproduce relatively rapidly also produce relatively large numbers of fruiting bodies (Simchen 1966; Xu 1995). Similarly, when the fungus Coprinus lagopus is grown on various media, the total dry weight of reproductive structures produced over 30 days is negatively correlated with the time taken to produce the first fruiting bodies (Madelin 1956), which is consistent with our model prediction that a relatively large fraction of available energy will be spent on reproduction when the timescale of reproduction is short.

\section{Discussion}

Species that grow on patchy, ephemeral substrates like fruit, phloem sap, or dung grow rapidly and reproduce over a relatively short timescale (Pugh 1980). Our model suggests that the relatively rapid growth of such species simply reflects the fact that nutrient-rich substrates that are easily digested can support higher rates of growth, particularly if the fungus is well adapted to growing on the substrate in question. This follows because, by definition, the embodied energy of the fungal biomass produced per unit time is equal to the amount of energy consumed per unit time, multiplied by the bioconversion efficiency (that is, joules grown per joule consumed). Both the rate of consumption and the maximal growth rate will be relatively high when the substrate is dense ( $\lambda$ is large), nutrient rich ( $\Omega$ is high), or easy to consume ( $\tau$ is short). If a patch of resource is present only for a brief window of time, then the timescale of reproduction for fungi that grow on that resource must be similarly brief. Our model implies that growth strategies that maximize the energy spent on reproduction over timescales that are similar to doubling time commit a large fraction of the available energy to reproduction. In contrast, when the timescale of reproduction is much longer than the doubling time, strategies that maximize the energy available for reproduction are very similar to strategies that maximize the growth rate, with only a small fraction of energy spent on reproduction (see fig. 5).

We also note that, independent of any details of our model, it is implausible that the bioconversion efficiency does not vary with the rate of growth. Growing very slowly will be inefficient because of the need to expend energy to sustain the existing colony while the new growth occurs. Conversely, there are inevitable additional costs associated with growing very fast, such as high requirements for transport and resource consumption. It is therefore reasonable to suppose that there is a growth rate $g_{\min }$ that minimizes the amount of energy spent while growing a unit volume. We consider it unlikely that any organism would grow slower than $g_{\min }$, because this would be an inefficient way of utilizing resources already captured as well as being a poor strategy for capturing additional resource.

Finally, we note that by recycling parts of the mycelium, the existing hyphal tips obtain an additional source of nutrients but that recycling will tend to reduce the fungus's capacity to synthesize and transport new cell constituents. Consequently, it is not obvious when recycling will be beneficial or why some species but not others recycle extensively. Our model implies that there is a relationship between the extent of recycling and the quality of resource on which the fungi grow. It suggests that biomass should be recycled only when fungi are growing on substrates that are patchy ( $\lambda$ is small), recalcitrant ( $\tau$ is long), or nutrient poor ( $\Omega$ is low). Furthermore, recycling is not beneficial if increasing the growth rate will significantly increase the amount of crowding ( $\Upsilon$ is large), so we expect to see extensive recycling only when the resource environment is challenging and the hyphae are not crowded (see fig. 2). Also note that in our model, maintaining hyphae that have digested the surrounding resource is beneficial only because it helps to reduce the cost of transport. In reality, there may be additional benefits to maintaining hyphae if, for example, a resource-depleted patch is refreshed by input of falling debris. This additional resource can be readily utilized only if the hyphae in that patch have not been recycled, and any such additional benefit associated with maintaining hyphae will serve to reduce the optimal amount of recycling relative to the levels predicted by our model. 
Our model unifies a diverse range of fungal phenomena, and its predictions for maximal growth rates and bioconversion efficiencies are consistent with data for growth on a range of different substrates. Further insights into optimal life-history strategies may be gained by extending our model to include competition between fungi for a finite pool of resource (Schmit 1999, 2002; Gilchrist et al. 2006) or additional metabolic costs arising from combative strategies, such as antibiotic production, mycoparasitism, contact inhibition, and mycelial lysis (Pugh 1980; Rayner and Todd 1980; Deacon 2005). A further refinement would be to model multiple resources, such as carbon and nitrogen, as reproductive structures typically require a larger concentration of nitrogen than does vegetative growth. Thus, in the case where nitrogen is limited, fungi may be forced to reduce the fraction of their energy budget that can be invested in reproduction (Plunkett 1953; Madelin 1956; Morton 1961). We also suspect that the inclusion of a second, limiting resource (e.g., nitrogen) may be needed to explain the evolution and persistence of different strategies for consuming the same substrate (Taylor and Williams 1974; Tilman 1990), such as the white-rot and brown-rot strategies for consuming wood (Deacon 2005).

\section{Acknowledgments}

L.L.M.H. and M.D.F. would like to thank the Human Frontiers Science Program, grant APRWJB0. We would also like to thank I. G. Johnston, A. Pringle, R. Sibly, L. Turnbull, and our anonymous reviewers for their helpful comments.

\section{Literature Cited}

Andrews, J. H. 1992. Fungal life-history strategies. Pages 119-145 in G. C. Carroll and D. T. Wicklow, eds. The fungal community: its organization and role in the ecosystem. 2nd ed. Dekker, New York.

Bebber, D. P., J. Hynes, P. R. Darrah, L. Boddy, and M. D. Fricker. 2007. Biological solutions to transport network design. Proceedings of the Royal Society B: Biological Sciences 274:2307-2315.

Boddy, L. 1999. Saprotrophic cord-forming fungi: meeting the challenge of heterogeneous environments. Mycologia 91:13-32.

Boddy, L., U. Büntgen, S. Egli, A. C. Gange, E. Heegaard, P. M. Kirk, A. Mohammad, and H. Kauserud. 2014. Climate variation effects on fungal fruiting. Fungal Ecology 10:20-33.

Boddy, L., J. C. Frankland, and P. van West, eds. 2008. Ecology of saprotrophic basidiomycetes. British Mycological Society Symposia Series, no. 28. Academic Press, London.

Boddy, L., J. Hynes, D. P. Bebber, and M. D. Fricker. 2009. Saprotrophic cord systems: dispersal mechanisms in space and time. Mycoscience 50:9-19.

Bolton, R. G., and L. Boddy. 1993. Characterization of the spatial aspects of foraging mycelial cord systems using fractal geometry. Mycological Research 97:762-768.

Deacon, J. 2005. Fungal biology. Blackwell, Malden.
Dighton, J. 2003. Fungi in ecosystem processes. Dekker, New York Dowson, C. G., A. D. M. Rayner, and L. Boddy. 1986. Outgrowth patterns of mycelial cord-forming basidiomycetes from and between woody resource units in soil. Journal of General Microbiology 132:203-211.

Falconer, R. E., J. L. Bown, N. A. White, and J. W. Crawford. 2005 Biomass recycling and the origin of phenotype in fungal mycelia. Proceedings of the Royal Society B: Biological Sciences 272:17271734.

Fathima, B. S., D. Abhinandan, B. S. Kumar, and B. R. Mohan. 2013. Mathematical modelling of an endophytic fungus Fusarium oxysporum NFX06 isolated from Nothapodytes foetida. International Journal of Chemistry and Chemical Engineering 3:123-130.

Fricker, M. D., L. Boddy, and D. P. Bebber. 2007. Network organisation of mycelial fungi. Pages 307-328 in R. J. Howard and N. A. R. Gow, eds. The mycota. VIII. Biology of the fungal cell. Springer, Berlin.

Gessner, M. O. 1997a. Fungal biomass, production and sporulation associated with particulate organic matter in streams. Limnetica 13 : 33-44.

. 1997b. Growth and production of aquatic hyphomycetes in decomposing leaf litter. Limnology and Oceanography 42:496-505.

Gilchrist, M. A., D. L. Sulsky, and A. Pringle. 2006. Identifying fitness and optimal life-history strategies for an asexual filamentous fungus. Evolution 60:970-979.

Hamilton, N. R. S., B. Schmid, and J. L. Harper. 1987. Life-history concepts and the population biology of clonal organisms. Proceedings of the Royal Society B: Biological Sciences 232:35-57.

Heaton, L. L. M., B. Obara, V. Grau, N. S. Jones, T. Nakagaki, L. Boddy, and M. D. Fricker. 2012a. Analysis of fungal networks. Fungal Biology Reviews 26:12-29.

Henn, M. R., G. Gleixner, and I. H. Chapela. 2002. Growth-dependent stable carbon isotope fractionation by basidiomycete fungi: $\delta^{13} \mathrm{C}$ pattern and physiological process. Applied and Environmental Microbiology 68:4956-4964.

Jones, H. L., and J. J. Worrall. 1995. Fungal biomass in decayed wood. Mycologia 87:459-466.

Josefsen, L., A. Droce, T. E. Sondergaard, J. L. Sørensen, J. Bormann, W. Schäfer, H. Giese, and S. Olsson. 2012. Autophagy provides nutrients for nonassimilating fungal structures and is necessary for plant colonization but not for infection in the necrotrophic plant pathogen Fusarium graminearum. Autophagy 8:326-337.

Knowles, J., P. Lehtovaara, M. Penttilä, T. Teeri, A. Harkki, and I. Salovuori. 1987. The cellulase genes of Trichoderma. Antonie van Leeuwenhoek Journal of Microbiology 53:335-341.

Kooijman, S. A. L. M. 2009. Dynamic energy budget theory for metabolic organisation. Cambridge University Press, Cambridge.

Lindahl, B. D., and S. Olsson. 2004. Fungal translocation - creating and responding to environmental heterogeneity. Mycologist 18: 79-88.

Madelin, M. F. 1956. Studies on the nutrition of Coprinus lagopus Fr., especially as affecting fruiting. Annals of Botany 20:307-330.

Money, N. 2008. Insights on the mechanics of hyphal growth. Fungal Biology Reviews 22:71-76.

Morton, A. G. 1961. The induction of sporulation in mould fungi. Proceedings of the Royal Society B: Biological Sciences 153:548-569.

Papagianni, M. 2004. Fungal morphology and metabolite production in submerged mycelial processes. Biotechnology Advances 22:189-259.

Plunkett, B. E. 1953. Nutritional and other aspects of fruit-body production in pure cultures of Collybia velutipes. Annals of Botany 17:193-218. 
Pollack, J. K., S. D. Harris, and M. R. Marten. 2009. Autophagy in filamentous fungi. Fungal Genetics and Biology 46:1-8.

Pringle, A., and J. W. Taylor. 2002. The fitness of filamentous fungi. Trends in Microbiology 10:474-481.

Pugh, G. J. F. 1980. Strategies in fungal ecology. Transactions of the British Mycological Society 75:1-14.

Rayner, A. D. M., and N. K. Todd. 1980. Population and community structure and dynamics of fungi in decaying wood. Advances in Botanical Research 7:333-420.

Ritz, K. 1995. Growth responses of some soil fungi to spatially heterogeneous nutrients. FEMS Microbiology Ecology 16:269-279.

Schmit, J. P. 1999. Resource consumption and competition by unit restricted fungal decomposers of patchy substrates. Oikos 87:509-519. 2002. Tradeoffs between reproduction and mycelium production in the unit-restricted decomposer Coprinus cinereus. Mycologia 94:40-48.

Shoji, J., and K. D. Craven. 2011. Autophagy in basal hyphal compartments: a green strategy of great recyclers. Fungal Biology Reviews 25:79-83.

Simchen, G. 1966. Fruiting and growth rates among dikaryotic progeny of single wild isolates of Schizophyllum commune. Genetics 53:1151-1165.

Sousa, T., T. Domingos, J.-C. Poggiale, and S. A. L. M. Kooijman. 2010. Dynamic energy budget theory restores coherence in biology. Philosophical Transactions of the Royal Society B: Biological Sciences 365:3413-3428.
Steinberg, G. 2006. Hyphal growth: a tale of motors, lipids, and the Spitzenkörper. Eukaryotic Cell 6:351-360.

Suberkropp, K. 1991. Relationships between growth and sporulation of aquatic hyphomycetes on decomposing leaf litter. Mycological Research 95:834-850.

Taylor, P. A., and P. J. B. Williams. 1974. Theoretical studies on the coexistence of competing species under continuous-flow conditions. Canadian Journal of Microbiology 21:90-98.

Tilman, D. 1990. Constraints and tradeoffs: toward a predictive theory of competition and succession. Oikos 58:3-15.

Trinci, A. J. P. 1971. Influence of the width of the peripheral growth zone on the radial growth rate of fungal colonies on solid media. Journal of General Microbiology 67:325-344.

Wessels, J. G. H. 1993. Wall growth, protein excretion and morphogenesis in fungi. New Phytologist 123:397-413.

Wu, J. Z., P. C. K. Cheung, K. H. Wong, and N. L. Huang. 2003. Studies on submerged fermentation of Pleurotus tuber-regium (Fr.) Singer-part 1: physical and chemical factors affecting the rate of mycelial growth and bioconversion efficiency. Food Chemistry $81: 389-393$.

Xu, J. 1995. Analysis of inbreeding depression in Agaricus bisporus. Genetics 141:137-145.

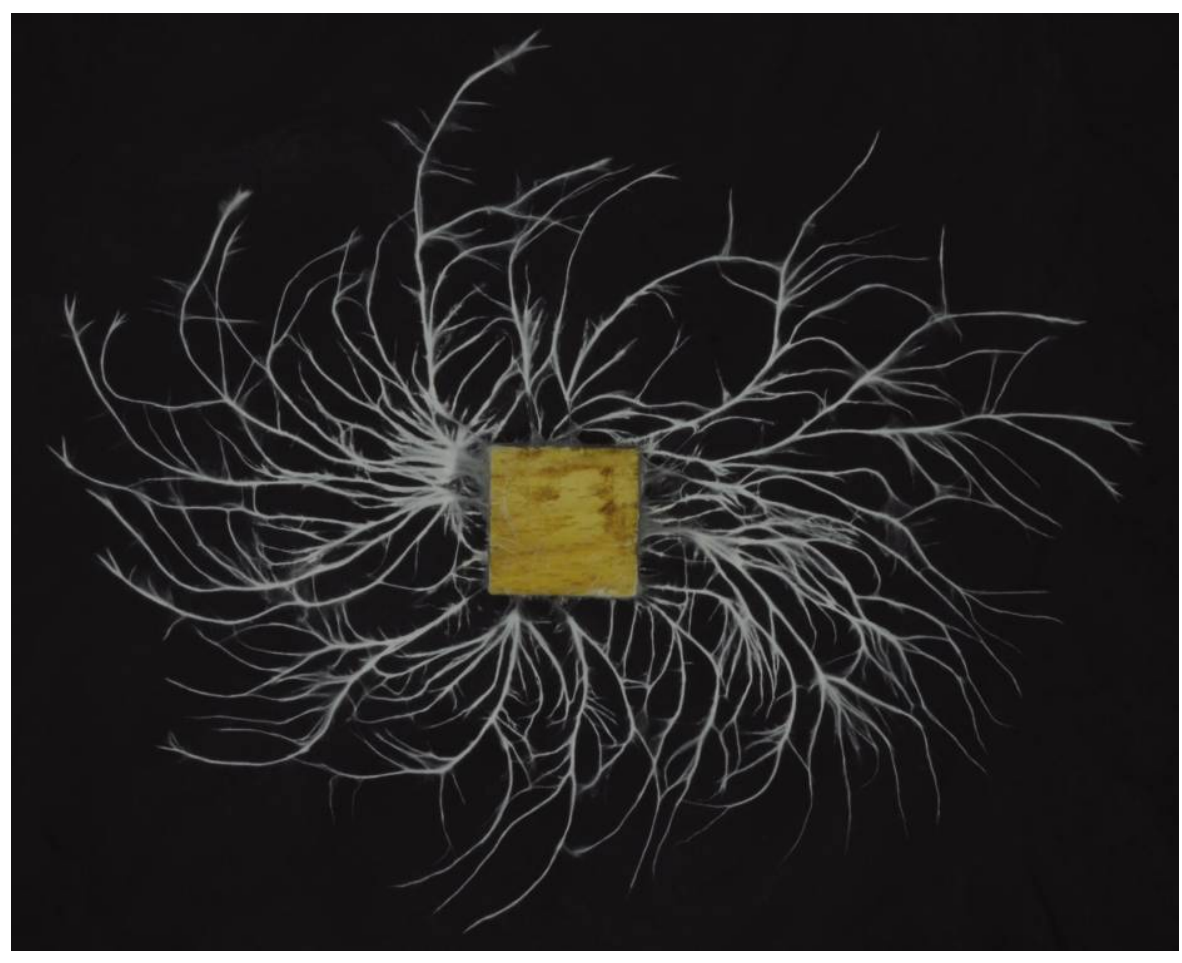

The cord-forming Basidiomycete Phanerochaete velutina growing out of a $2 \mathrm{~cm} \times 2 \mathrm{~cm}$ woodblock, in search of more wood to digest. Photograph by M. D. Fricker. 